\title{
Adipositastherapie
}

\section{Eine Leistung für die gynäkologische Praxis}

\author{
Neben den bekannten kardiologischen, metabolischen und orthopädischen Folgeerkrankungen rücken \\ zunehmend auch die Zusammenhänge zwischen Übergewicht und gynäkologischen Erkrankungen in \\ den Blickpunkt. Für den Frauenarzt ist es daher eine immer wichtigere Aufgabe, in seiner Praxis das \\ Problem Übergewicht und Adipositas therapeutisch anzugehen.
}

$\mathrm{D}$ ie "globale Adipositas-Epidemie“ geht auch an Deutschland nicht vorbei. Im Gegenteil: Nach den Erhebungen des Mikrozensus 2009 sind inzwischen $41,4 \%$ aller Deutschen (60,1\% Männer, 42,9\% Frauen) übergewichtig bzw. adipös. Die Bundesrepublik nimmt damit europaweit einen Spitzenplatz ein [1].

Studienergebnisse belegen, dass Adipositas die Fertilität deutlich reduziert [2]. Während der Schwangerschaft erhöht sich durch die Adipositas sowohl das Risiko für mütterliche Komplikationen [3] als auch für kindliche Missbildungen [4]. Auch die Rate operativer Entbindungen steigt signifikant [5]. Durch die endokrine Aktivität des Fettgewebes steigt darüber hinaus bei Übergewicht die Rate hormonabhängiger Tumoren [6, 7]. Insbesondere Veränderungen des IGF-1-Metabolismus und die Induktion eines chronisch inflammatorischen Milieus begünstigen Übergewicht, aber auch die Entstehung von Malignomen, die nicht steroidhormonabhängig sind [8].

Der Ernährungsberatung und der Adipositastherapie kommt daher in der gynäkologischen Praxis eine verstärkte Bedeutung zu. Als effektive Maßnahme hat sich dabei in den letzten Jahren der Einsatz standardisierter Formula-Diäten etabliert [9]. Hier gibt es inzwischen eine Vielzahl kommerzieller Gewichtsreduktionsprogramme, die in der Praxis Anwendung finden (Optifast ${ }^{\circ}, \mathrm{BCM}^{\circ}$, FormMed $^{\oplus}$, Bodymed ${ }^{\oplus}$, etc.). All diese Programme verknüpfen die Gewichtsreduktion mittels Formula-Diät mit einem gezielten Verhaltenstraining, denn - das zeigt die Studienlage eindeutig - nur im Zusammenspiel mit einer intensiven Ernährungsberatung und Verhaltensthera-

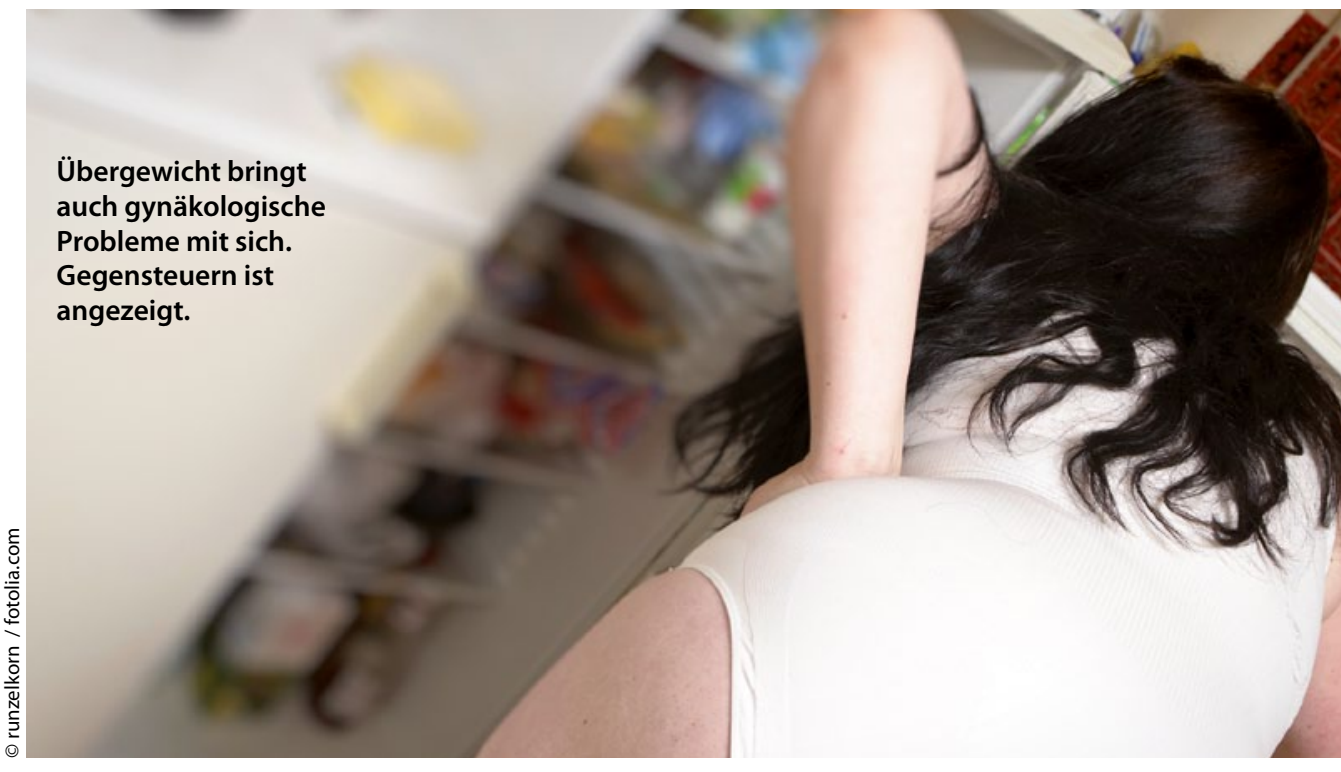

pie lässt sich eine nachhaltige Gewichtsreduktion erzielen und das Gewicht nachfolgend halten [10].

\section{Beratung auf der Höhe der Zeit}

Genau hier jedoch liegt das Problem für den praktisch tätigen Frauenarzt. Denn Beratung und Verhaltensmodifikationen sind zeitintensive Maßnahmen. Und Zeit ist in der ärztlichen Praxis bekanntlich eine knappe Ressource. Ein häufig gewählter Ausweg aus diesem Dilemma besteht in der Kooperation mit einer Ernährungsberaterin oder Ökotrophologin, die den zeitintensiven Beratungsteil übernehmen kann. Im Zeitalter fortgeschrittener Informationstechnologien bietet sich inzwischen jedoch eine weitere Option an: Die Verlegung der Ernährungsberatung und des Verhaltenstrainings in das Internet.

Neuere Studien zeigen, dass - zumindest für diejenigen Anwender, die mit diesem Medium umgehen können - webbasierte
Schulungen der klassischen „Face-to-FaceBeratung" sogar überlegen sind [11, 12]. Die Teilnehmer sind nicht mehr an fixe Sprechzeiten gebunden und können zeitlich unabhängig die entsprechenden Schulungen zu Hause oder an einem Ort ihrer Wahl nutzen. In Deutschland ist das erste Programm, das auf einer derartigen Kombination von Reduktionsdiät und internetbasierter Ernährungsberatung beruht, im März 2012 von der Firma NSA unter dem Namen Juice Plus Shape ${ }^{\circledR}$ eingeführt worden. Vor allem für den niedergelassenen Arzt eröffnet sich damit eine interessante Perspektive, Adipositastherapie ohne großen Zeitaufwand und dennoch effektiv in der eigenen Praxis anzubieten.

\section{Literatur beim Verfasser}

Prof. Dr. med. Bernd Kleine-Gunk

Euromed Clinic Fürth

Europaalle 1, 90763 Fürth 